\title{
Sequential therapy with ramucirumab and/or checkpoint inhibitors for non-small-cell lung cancer in routine practice
}

Cliff Molife ${ }^{* 1}{ }^{1}$, Lisa M Hess ${ }^{1}$, Zhanglin Lin Cui ${ }^{1}$, Xiaohong Ivy Li ${ }^{1}$, Julie Beyrer ${ }^{1}$, Malika Mahoui ${ }^{1} \&$ Ana B Oton ${ }^{1}$

${ }^{1}$ Eli Lilly \& Company, Lilly Corporate Center, Indianapolis, IN 46285, USA

*Author for correspondence: Tel.: +1 317220 7074; molife_cliff@lilly.com

\begin{abstract}
Aim: To describe treatment patterns and outcomes for advanced/metastatic non-small-cell lung cancer (aNSCLC) treated with single-agent or combination ramucirumab (ramucirumab-based) and/or immune checkpoint inhibitor (ICl-based) therapy. Materials \& methods: Retrospective study of aNSCLC patients $(n=4054)$ identified in the Flatiron Health database, who received at least two treatment lines including ramucirumab- and/or ICl-based regimens between December 2014 and May 2017. Results: Median overall survival $(95 \% \mathrm{Cl})$ from aNSCLC diagnosis was 29.3 (25.5-33.0) months for patients receiving sequential ramucirumab- and ICI-based therapy $(n=245), 15.1(12.6-18.2)$ months for patients receiving sequences including ramucirumab- without ICl-based therapy $(n=112)$, and $23.1(21.9-24.2)$ months for patients receiving ICl-based therapy without ramucirumab-based therapy in sequence $(n=3697)$. Conclusion: Results provide real-world survival estimates for aNSCLC treated with sequences including ramucirumab- and/or $\mathrm{ICI}$-based therapies.
\end{abstract}

First draft submitted: 22 November 2018; Accepted for publication: 23 January 2019; Published online: 22 February 2019

Keywords: atezolizumab $\bullet$ immune checkpoint inhibitor $\bullet$ nivolumab $\bullet$ non-small-cell lung cancer $\bullet$ overall survival - pembrolizumab • ramucirumab • treatment sequence

Lung cancer is the leading cause of cancer-related deaths in the USA, with 234,030 new cases and 154,050 deaths estimated in 2018 alone, accounting for about $25 \%$ of all cancer deaths [1]. Non-small-cell lung cancer (NSCLC) accounts for about $80-85 \%$ of all lung cancers [2,3] of which squamous cell carcinoma $(\sim 25-30 \%)$ and nonsquamous carcinoma (including adenocarcinoma, large-cell carcinoma, and other specified carcinomas; $\sim 70-75 \%)$ are the two predominant histologic types [2,3]. The majority (79\%) of NSCLC cases are diagnosed at later stages, and for patients with metastatic disease, the 5 -year relative survival rate is $5 \%[1,3]$.

For patients with advanced or metastatic NSCLC (aNSCLC) lacking actionable genetic mutations, platinumbased chemotherapy has been the standard frontline treatment and the newly emerging standard of care in this setting is immune checkpoint inhibitors as single agents (i.e., pembrolizumab) or in combination with platinum-based chemotherapy (including pembrolizumab or atezolizumab in combination with platinum-based chemotherapy) [4-10]. Although many patients with aNSCLC respond to initial therapy, all patients eventually experience disease progression on or after treatment [11] and about half $(47-51 \%)$ of them receive subsequent treatment in a real-life setting [12-14]. When initial platinum-based therapy fails, current American Society of Clinical Oncology and National Comprehensive Cancer Network recommendations are for second-line (2L) treatment with immune checkpoint inhibitors (immune checkpoint inhibitors [ICIs], if not given in first-line [1L]), single-agent chemotherapy or an antiangiogenic agent (i.e., ramucirumab) plus docetaxel $[4,5]$. These guidelines reflect US FDA approvals of ramucirumab + docetaxel in December 2014 [15], nivolumab in March [16] and October 2015 [17], pembrolizumab in October 2015 [18] and atezolizumab in October 2016 [19], based on findings from several randomized trials showing significant survival benefits with these regimens over single-agent docetaxel in the $2 \mathrm{~L}$ treatment setting for aNSCLC [20-24]. Response rates from these trials were 14-20\% for ICIs (irrespective of 
PD-L1 expression levels) and 23\% for ramucirumab + docetaxel (all histologies); median overall survival (OS) was 9.2-13.8 months and 10.5 months, and 1-year survival rates were 42-55 and 43\%, respectively [20-24].

Although clinical trials have demonstrated statistically significant improvements in OS with ramucirumab + docetaxel or ICIs compared with single-agent docetaxel [20-24], there is limited evidence regarding the use of these agents in community oncology practice settings, and the effectiveness of sequencing strategies including these agents remains unknown. Therefore, this study was designed to describe treatment patterns and survival outcomes among patients diagnosed with aNSCLC treated with regimens containing ramucirumab, nivolumab, pembrolizumab or atezolizumab in oncology practices across the USA.

\section{Materials \& methods}

\section{Data source}

This retrospective observational study used electronic health record data from the aNSCLC cohort of the Flatiron Health database, which includes a geographically diverse sample of over 36,000 patients (as of September 2017) with aNSCLC at community and academic cancer centers in the Flatiron network across the USA [25]. While the database predominantly represents the community setting, it is nationally illustrative of age, gender and geographic location and represents approximately 2500 clinicians, 265 cancer clinics, 800 different sites of care and 2 million active cancer patients. The database is refreshed monthly and enables a longitudinal view of structured and unstructured electronic health record data for cancer patients treated in the Flatiron network. Structured data include data points that are organized in a predefined manner such as patient demographics, laboratory values and medication administrations. Unstructured data are not organized in a predefined manner (e.g., diagnoses and staging from physician notes and pathology reports) and are curated through technology-enabled abstraction by trained abstractors at Flatiron. Patients in the Flatiron database have been diagnosed with Stage IIIB or IV NSCLC or presented with earlier stage NSCLC but subsequently experienced disease progression or recurrence, with two or more documented visits, on or after 1st January 2011. This noninterventional study does not qualify as human subjects research in accordance with the US Code of Federal Regulations (CFR), 45 CFR 46.102(f), and is thereby exempt from Institutional Review Board evaluation.

\section{Patient selection}

Adult ( $\geq 18$ years) patients treated with at least two lines of therapy who received systemic treatment containing ramucirumab (ramucirumab-based therapy) and/or containing pembrolizumab, nivolumab or atezolizumab (ICIbased therapy) in any line of therapy for aNSCLC between 12 December 2014 and 31 May 2017 were identified in the Flatiron database and assigned to one of three study cohorts based on treatment sequences. This study time frame spans a period when key changes have occurred in the postprogression treatment setting for aNSCLC. The Ram and ICI cohorts included patients who received regimens containing either ramucirumab- or ICIbased therapy, respectively, but not both, whereas the Ram $\leftrightarrow$ ICI cohort included patients who received both ramucirumab- and ICI-based therapy in any sequence of care. Cohort assignments were further stratified by the timing of ramucirumab-based therapy relative to ICI-based therapy in the sequence of care (Ram pre-ICI and Ram post-ICI) for the Ram $\leftrightarrow$ ICI cohort, by histologic subtype (squamous and nonsquamous), and by duration of $1 \mathrm{~L}$ treatment ( $\leq 12$ weeks and $>12$ weeks). A 12-week cutoff was used as a proxy for rapid disease progression status as clinical trial data have demonstrated poorer prognosis in patients with more rapid than typical disease progression during $1 \mathrm{~L}$ therapy, including those who discontinue $1 \mathrm{~L}$ treatment after a short ( $\leq 12$ weeks) duration [26]. Eligible patients were followed until the last recorded visit date (loss to follow-up), date of death or the latest date available at the time of the study (31st August 2017), whichever was earliest.

\section{Study variables}

Baseline demographic (including age, gender, race, smoking status, weight) and clinical (including stage, metastatic recurrence, histologic type, biomarker status) characteristics and the name, administration route and dosage of anticancer therapy were obtained from the database. Treatment duration was defined as the time between the first and the last infusion of the regimen during a line of therapy. As available, Eastern Cooperative Oncology Group performance status (ECOG PS) and biomarker testing results for EGFR or KRAS mutations, ALK or ROS1 rearrangements or PD-L1 expression were reported. The date (month and year) of death was obtained from the database. The OS was defined as the time-period from the date of aNSCLC diagnosis until death. 


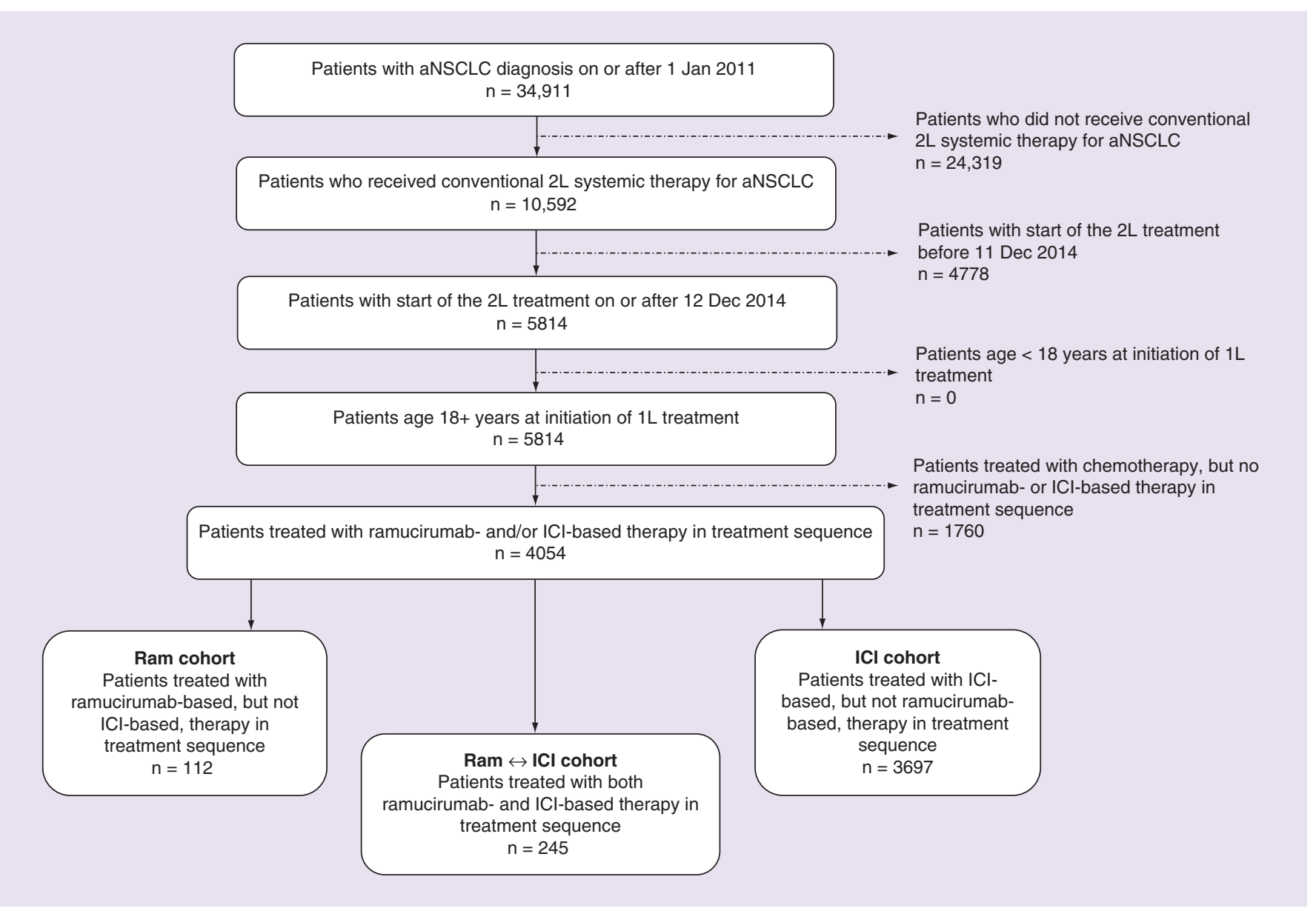

Figure 1. Patient attrition.

1L: First-line; 2L: Second-line; aNSCLC: Advanced or metastatic non-small-cell lung cancer; ICI: Immune checkpoint inhibitor (nivolumab, pembrolizumab, atezolizumab); ICI-based therapy: Monotherapy or combination regimen containing an ICl; Ramucirumab-based therapy: Monotherapy or combination regimen containing ramucirumab.

\section{Statistical analysis}

Descriptive statistics such as frequency and percentage for categorical variables and mean, standard deviation, and median for continuous variables were used to summarize the demographic and clinical characteristics as well as treatment patterns, including sequences, number of infusions (cycles) and treatment duration (in days). The Kaplan-Meier method was used to estimate survival curves, medians and associated 95\% CIs. Patients, who were lost to follow-up or who reached the end of the study period without evidence of death, were censored at their last activity date (the latest of any drug administration or visit date) for OS analysis. Given the descriptive, noncomparative design of this study, no formal sample size and power calculations were conducted and no specific hypotheses were tested. All statistical analyses were performed using SAS version 9.3 software (SAS Institute Inc., NC, USA).

\section{Results}

\section{Patients}

In total, 4054 patients were eligible for the study. Ram $\leftrightarrow$ ICI, Ram and ICI treatment cohorts (as defined previously) were comprised of 245 (6\%), 112 (3\%) and 3697 (91\%) patients, respectively (Figure 1). Baseline patient characteristics are summarized in Table 1 for each cohort and by histology. The median age (range) was 63 (40-84), 67 (41-83) and 68 (21-85) years, respectively. The majority of the Ram $\leftrightarrow$ ICI cohort $(56 \%)$ were aged $<65$ years, whereas the majority of the Ram (60\%) and ICI (63\%) cohorts were aged $\geq 65$ years. Among the Ram $\leftrightarrow$ ICI, Ram and ICI cohorts, the proportions of patients with nonsquamous histology were 72,80 and $67 \%$, 


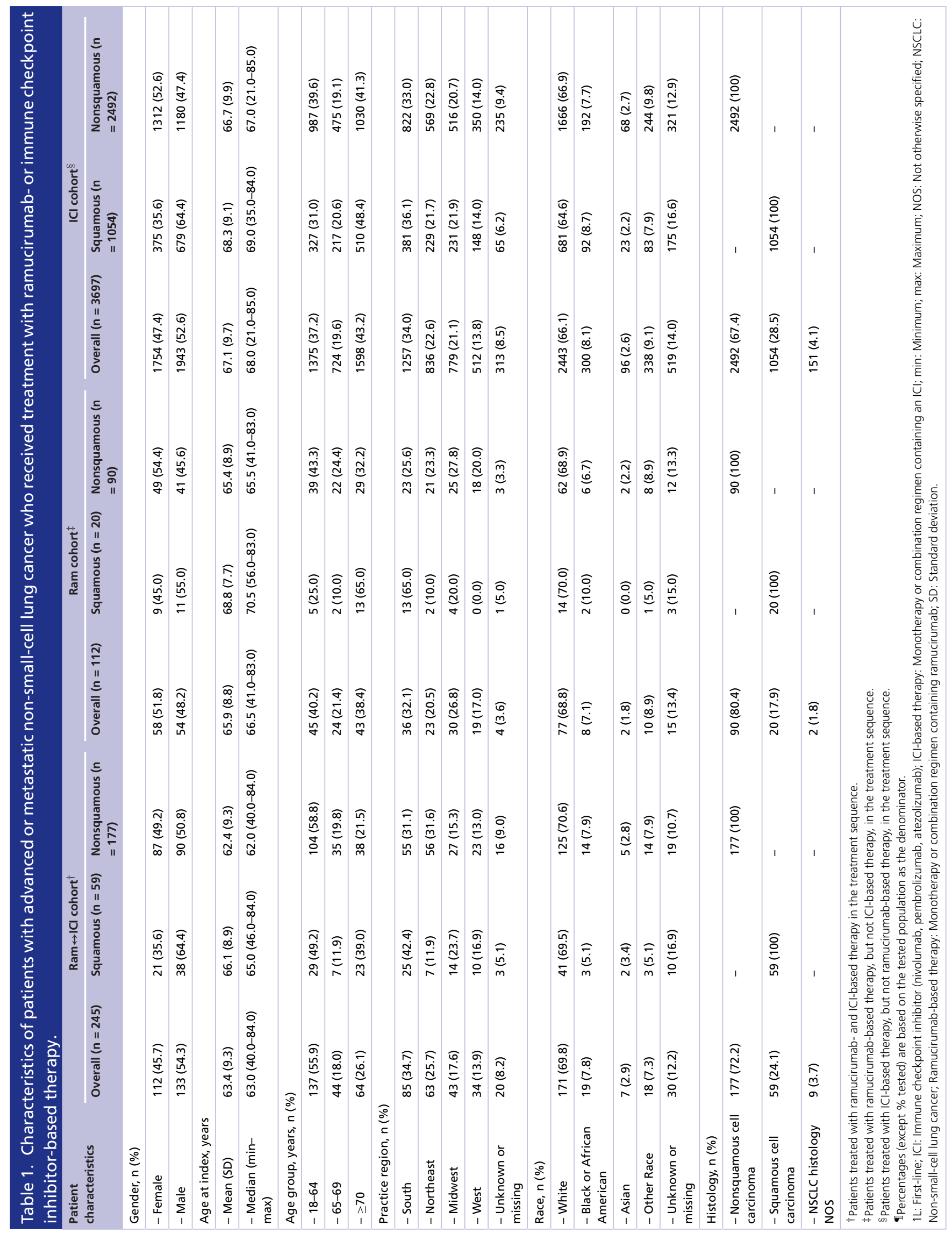




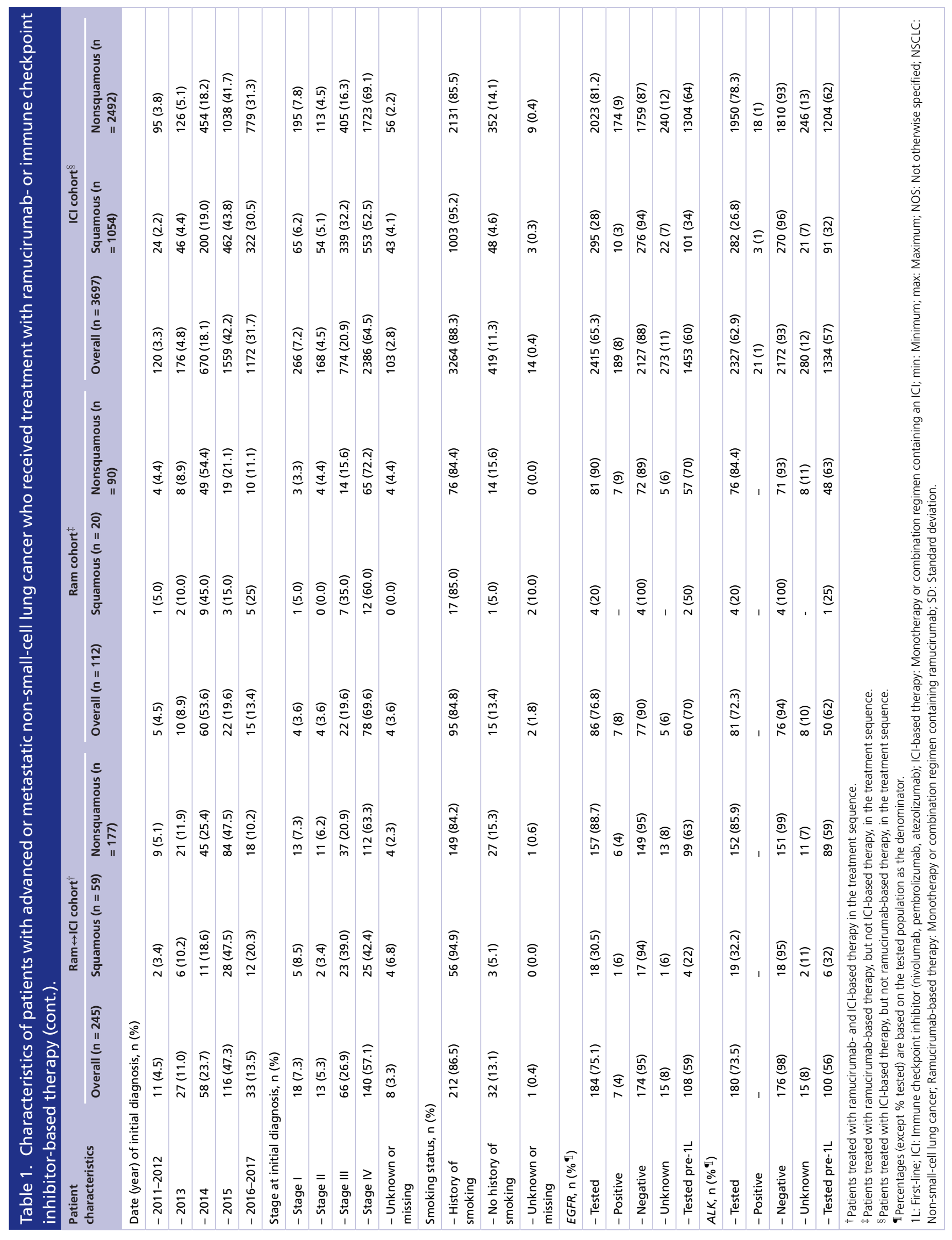




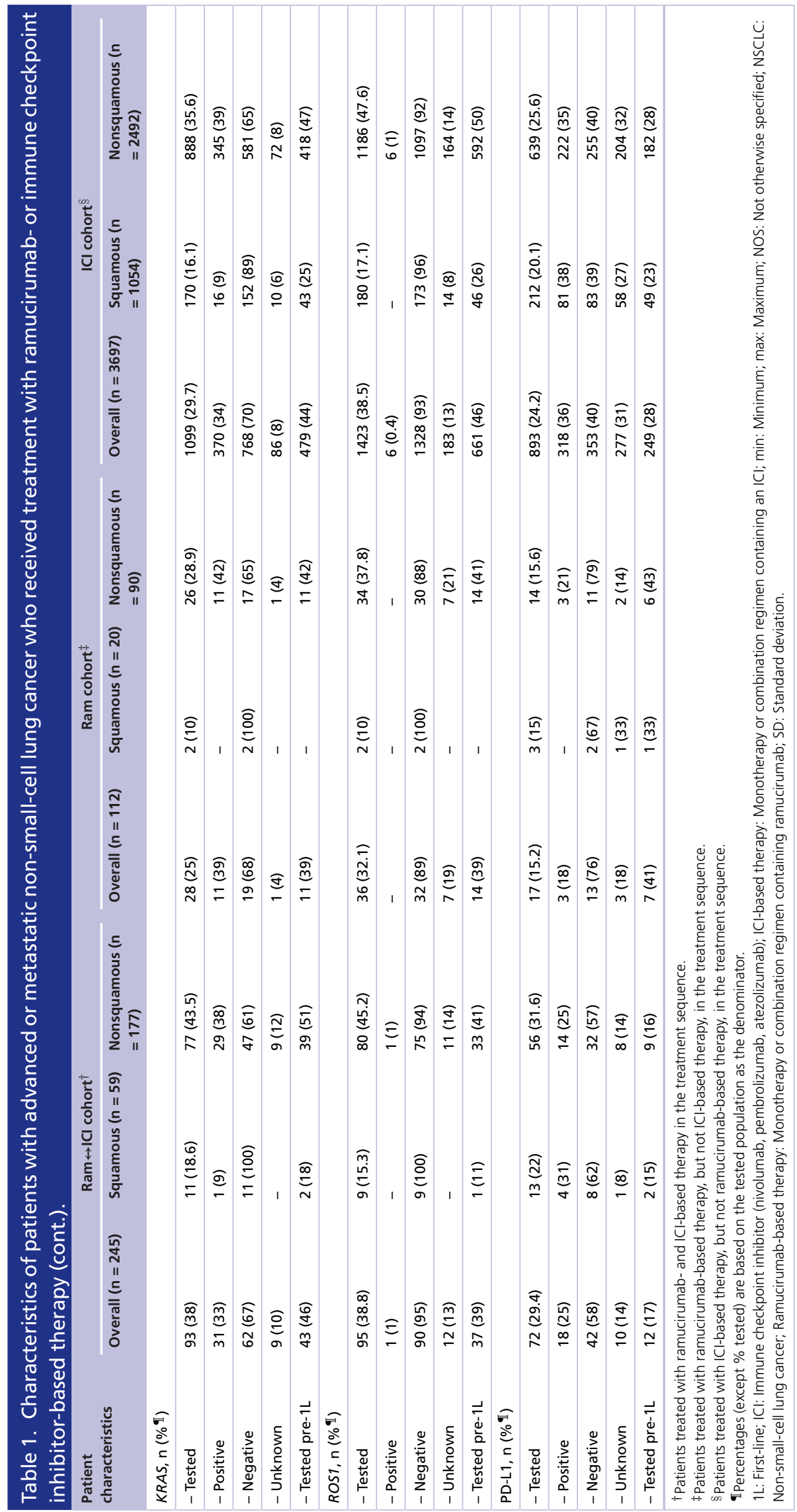


respectively, and 57, 70 and 65\% had an initial diagnosis of Stage IV disease. These results were generally similar by histology type. Baseline ECOG PS was missing for the vast majority ( $>80 \%)$ of patients.

Overall, most patients were tested for $E G F R$ mutation or $A L K$ translocation but not for KRAS mutation, ROS1 rearrangement or PD-L1 expression (Table 1). Of those tested, the majority tested negative for EGFR or KRAS mutations and $A L K$ or ROS1 translocations. Among the 29, 15 and 24\% of the Ram $\leftrightarrow$ ICI, Ram and ICI cohorts tested for PD-L1 expression, respectively, 25, 18 and 36\% were PD-L1 'positive'. Most patients were tested for biomarkers prior to $1 \mathrm{~L}$ treatment and testing rates were generally lower for the squamous group (Table 1).

\section{Treatment patterns}

Combinations

Although ramucirumab was used in combination with docetaxel among $90 \%$ of patients, consistent with the US FDA-approved label [27], in about $10 \%$ of patients, ramucirumab was used as monotherapy or in combination with other single-agent or doublet chemotherapy regimens, ICIs, tyrosine kinase inhibitors or bevacizumab (Table 2). Overall, 24 distinct regimens containing ramucirumab were observed. Similarly, numerous $(>50)$ distinct combinations including ICIs were observed although ICIs were most frequently used as monotherapy, consistent with the US FDA labels for these agents (Table 2) [28-30] . The components of ramucirumab or ICI combination regimens varied across lines of therapy (Table 2), and to a lesser extent, across histology (data not shown). For example, the number of distinct combination regimens including ramucirumab or ICIs was generally higher among patients with nonsquamous disease and in the postprogression setting, consistent with the higher use of ramucirumab- and ICI-based therapy observed in these settings.

\section{Usage trends}

Figure 2 shows the general trend in usage of ramucirumab- and ICI-based therapy during the study period. Initiation of ramucirumab-based therapy, especially in $2 \mathrm{~L}$, was the highest in quarter (Q) 12015 , after which ICI-based therapy increased rapidly, peaking in Q1 2016. Nivolumab was the most frequently used ICI since Q1 2015, followed by pembrolizumab starting in Q4 2015 and then atezolizumab starting in Q4 2016. Use of ramucirumab-based therapy in sequence with ICI-based therapy, particularly in third-line (3L) following $2 \mathrm{~L} \mathrm{ICI}$ therapy, increased steadily from Q1 2015 and peaked in Q4 2016.

\section{Sequences}

Overall, there were 235 distinct sequencing strategies including ramucirumab-based therapy and 1219 distinct treatment sequences including ICI-based therapy. Figure 3 illustrates the ten most common sequences including ramucirumab- or ICI-based therapy, respectively. Most Ram $\leftrightarrow$ ICI patients $(86 \%)$ received at least three lines of therapy while most of the Ram cohort $(63 \%)$ and ICI cohort (63\%) received two treatment lines. Among patients treated with ramucirumab-based therapy $(\mathrm{n}=357)$ or ICI-based therapy $(\mathrm{n}=3942)$ overall, the most common $1 \mathrm{~L}$ regimens were carboplatin-based regimens (38 and $74 \%$, respectively) with or without pemetrexed, bevacizumab and/or a taxane; followed by single-agent nivolumab (8 and 6\%, respectively). Ramucirumab- and ICI-based regimens were most often initiated in $2 \mathrm{~L}$ ( 42 and $73 \%$, respectively), with 7 and $6 \%$ receiving these regimens in $1 \mathrm{~L}$ and 36 and $20 \%$ in $3 \mathrm{~L}$, respectively. Of patients treated with ramucirumab-based $(\mathrm{n}=151)$ or ICI-based $(\mathrm{n}=2858)$ regimens in 2L, $64(42 \%)$ and $666(23 \%)$, respectively, went on to receive subsequent treatment $(3 \mathrm{~L}+)$.

Overall, ramucirumab-based therapy was most often $(n=245,69 \%)$ used in sequence with an ICI-based regimen, and of these patients, ramucirumab-based therapy was more frequently (160 patients, 65\%) used after ICI-based therapy, particularly nivolumab-containing regimens. Among the 160 patients receiving ramucirumab-following ICI-based therapy, 31 (19\%) received ICI-based therapy in 1L, and of those, $22(71 \%)$ received ramucirumabbased therapy in $2 \mathrm{~L}$. For the Ram cohort, prior or subsequent lines of therapy without ramucirumab-based therapy mostly contained carboplatin-based regimens. Overall, ICI-based regimens were most frequently used in sequential lines before or following single-agent or combination chemotherapy. Some patients treated with ramucirumab- or ICI-based therapy received the same or a different ramucirumab- or ICI-based regimen, or a targeted agent, in prior or future lines of therapy (Figure 3).

\section{Treatment duration}

Table 3 shows dosing and treatment duration with ramucirumab or ICI therapy. Overall, patients received a median of three doses of ramucirumab (median dose $10 \mathrm{mg} / \mathrm{kg}$ ), six doses of nivolumab (median dose $3 \mathrm{mg} / \mathrm{kg}$ ), three 
Table 2. Ramucirumab- or ICl-based therapy for patients with advanced or metastatic non-small-cell lung cancer.

\begin{tabular}{|c|c|c|c|c|c|c|c|}
\hline$\#$ & Regimen & All lines $n(\%)$ & $1 \mathrm{~L}(\mathrm{~N})$ & $2 \mathrm{~L}(\mathrm{~N})$ & $3 L(N)$ & $4 \mathrm{~L}(\mathrm{~N})$ & $5 \mathrm{~L}(\mathrm{~N})$ \\
\hline \multicolumn{8}{|c|}{ Ramucirumab-based regimens } \\
\hline 1. & Ramucirumab + docetaxel & $320(86.25)$ & 19 & 135 & 115 & 38 & 13 \\
\hline 2. & Ramucirumab & $8(2.16)$ & 3 & 4 & 1 & - & - \\
\hline 3. & Ramucirumab + paclitaxel & $13(3.50)$ & 2 & 3 & 4 & 3 & 1 \\
\hline 4. & Ramucirumab + nivolumab + pemetrexed & $1(0.27)$ & 1 & - & - & - & - \\
\hline 5. & Ramucirumab + gemcitabine & $3(0.81)$ & - & 2 & - & 1 & - \\
\hline 6. & Ramucirumab + docetaxel + erlotinib & $2(0.54)$ & - & 1 & - & 1 & - \\
\hline 7. & Ramucirumab + bevacizumab + paclitaxel protein-bound & $1(0.27)$ & - & 1 & - & - & - \\
\hline 8. & Ramucirumab + nivolumab + pembrolizumab + pemetrexed & $1(0.27)$ & - & 1 & - & - & - \\
\hline 9. & Ramucirumab + paclitaxel protein-bound & $6(1.62)$ & - & 1 & 2 & 3 & - \\
\hline 10. & Ramucirumab + carboplatin + paclitaxel & $2(0.54)$ & - & 1 & - & 1 & - \\
\hline 11. & Ramucirumab + cisplatin + paclitaxel & $1(0.27)$ & - & 1 & - & - & - \\
\hline 12. & Ramucirumab + carboplatin + paclitaxel protein-bound & $1(0.27)$ & - & 1 & - & - & - \\
\hline 13. & Ramucirumab + bevacizumab + docetaxel & $1(0.27)$ & - & - & 1 & - & - \\
\hline 14. & Ramucirumab + docetaxel + nivolumab & $1(0.27)$ & - & - & 1 & - & - \\
\hline 15. & Ramucirumab + docetaxel + pemetrexed & $1(0.27)$ & - & - & 1 & - & - \\
\hline 16. & Ramucirumab + afatinib + docetaxel + erlotinib & $1(0.27)$ & - & - & 1 & - & - \\
\hline 17. & Ramucirumab + afatinib + paclitaxel protein-bound & $1(0.27)$ & - & - & 1 & - & - \\
\hline 18. & Ramucirumab + docetaxel + pembrolizumab & $1(0.27)$ & - & - & - & 1 & - \\
\hline 19. & Ramucirumab + cisplatin + docetaxel + gemcitabine & $1(0.27)$ & - & - & - & 1 & - \\
\hline 20. & Ramucirumab + cisplatin + gemcitabine & $1(0.27)$ & - & - & - & 1 & - \\
\hline 21. & Ramucirumab + pemetrexed & $1(0.27)$ & - & - & - & 1 & - \\
\hline 22. & Ramucirumab + docetaxel + gemcitabine & $1(0.27)$ & - & - & - & 1 & - \\
\hline 23. & Ramucirumab + vinorelbine & $1(0.27)$ & - & - & - & 1 & - \\
\hline 24. & Ramucirumab + cisplatin + dabrafenib + trametinib & $1(0.27)$ & - & - & - & - & 1 \\
\hline & umab-based regimens & $371(100.00)$ & 25 & 151 & 127 & 53 & 15 \\
\hline \multicolumn{8}{|c|}{ Nivolumab-based regimens } \\
\hline 1. & Nivolumab & $3533(96.66)$ & 218 & 2487 & 666 & 135 & 27 \\
\hline 2. & Nivolumab + paclitaxel protein-bound & $11(0.30)$ & 1 & 5 & 3 & 1 & 1 \\
\hline 3. & Nivolumab + pemetrexed + ramucirumab & $1(0.03)$ & 1 & - & - & - & - \\
\hline 4. & Nivolumab + ipilimumab & $10(0.27)$ & 1 & 6 & 1 & 2 & - \\
\hline 5. & Nivolumab + pemetrexed & $8(0.22)$ & - & 7 & 1 & - & - \\
\hline 6. & Nivolumab + carboplatin + paclitaxel & $6(0.16)$ & - & 5 & 1 & - & - \\
\hline 7. & Nivolumab + erlotinib & $15(0.41)$ & - & 5 & 6 & 4 & - \\
\hline 8. & Nivolumab + clinical study drug & $3(0.08)$ & - & 3 & - & - & - \\
\hline 9. & Nivolumab + docetaxel & $5(0.14)$ & - & 3 & 2 & - & - \\
\hline 10. & Nivolumab + carboplatin + paclitaxel protein-bound & $3(0.08)$ & - & 3 & - & - & - \\
\hline 11. & Nivolumab + bevacizumab + pemetrexed & $4(0.11)$ & - & 3 & 1 & - & - \\
\hline 12. & Nivolumab + afatinib & $5(0.14)$ & - & 3 & 1 & - & 1 \\
\hline 13. & Nivolumab + carboplatin + gemcitabine & $4(0.11)$ & - & 3 & 1 & - & - \\
\hline 14. & Nivolumab + bevacizumab + carboplatin + pemetrexed & $3(0.08)$ & - & 2 & 1 & - & - \\
\hline 15. & Nivolumab + crizotinib & $4(0.11)$ & - & 2 & 1 & 1 & - \\
\hline 16. & Nivolumab + gemcitabine & $5(0.14)$ & - & 2 & 1 & 2 & - \\
\hline 17. & Nivolumab + cisplatin + gemcitabine & $2(0.05)$ & - & 2 & - & - & - \\
\hline 18. & Nivolumab + paclitaxel & $6(0.16)$ & - & 2 & 3 & 1 & - \\
\hline 19. & Nivolumab + gemcitabine + vinorelbine & $2(0.05)$ & - & 1 & - & 1 & - \\
\hline 20. & Nivolumab + carboplatin + docetaxel & $1(0.03)$ & - & 1 & - & - & - \\
\hline 21. & Nivolumab + pembrolizumab + pemetrexed + ramucirumab & $1(0.03)$ & - & 1 & - & - & - \\
\hline 22. & Nivolumab + docetaxel + gemcitabine & $1(0.03)$ & - & 1 & - & - & - \\
\hline
\end{tabular}


Table 2. Ramucirumab- or ICl-based therapy for patients with advanced or metastatic non-small-cell lung cancer (cont.).

\begin{tabular}{|c|c|c|c|c|c|c|c|}
\hline$\#$ & Regimen & All lines n (\%) & $1 \mathrm{~L}(\mathrm{~N})$ & $2 \mathrm{~L}(\mathrm{~N})$ & $3 L(N)$ & $4 L(N)$ & $5 L(N)$ \\
\hline 23. & Nivolumab + carboplatin + pemetrexed & $2(0.05)$ & - & 1 & 1 & - & - \\
\hline 24. & Nivolumab + bevacizumab & $2(0.05)$ & - & 1 & 1 & - & - \\
\hline 25. & Nivolumab + carboplatin & $1(0.03)$ & - & 1 & - & - & - \\
\hline 26. & Nivolumab + carboplatin + Etoposide & $1(0.03)$ & - & 1 & - & - & - \\
\hline 27. & Nivolumab + osimertinib & $3(0.08)$ & - & - & 3 & - & - \\
\hline 28. & Nivolumab + gefitinib & $3(0.08)$ & - & - & 2 & 1 & - \\
\hline 29. & Nivolumab + docetaxel + ramucirumab & $1(0.03)$ & - & - & 1 & - & - \\
\hline 30. & Nivolumab + Vinorelbine & $2(0.05)$ & - & - & 1 & 1 & - \\
\hline 31. & Nivolumab + carboplatin + docetaxel & $1(0.03)$ & - & - & 1 & - & - \\
\hline 32. & Nivolumab + cisplatin + gemcitabine & $1(0.03)$ & - & - & - & 1 & - \\
\hline 33. & Nivolumab + erlotinib + osimertinib & $1(0.03)$ & - & - & - & 1 & - \\
\hline 34. & Nivolumab + bevacizumab + carboplatin + pemetrexed & $1(0.03)$ & - & - & - & 1 & - \\
\hline 35. & Nivolumab + pembrolizumab & $1(0.03)$ & - & - & - & 1 & - \\
\hline 36. & Nivolumab + ipilimumab + vinorelbine & $1(0.03)$ & - & - & - & - & 1 \\
\hline 37. & Nivolumab + alectinib + ceritinib & $1(0.03)$ & - & - & - & - & 1 \\
\hline \multicolumn{2}{|c|}{ All nivolumab-based regimens } & $3655(100.00)$ & 221 & 2551 & 699 & 153 & 31 \\
\hline \multicolumn{8}{|c|}{ Pembrolizumab-based regimens } \\
\hline 1. & Pembrolizumab & $354(95.68 \%)$ & 30 & 230 & 67 & 19 & 8 \\
\hline 2. & Pembrolizumab + carboplatin + pemetrexed & $3(0.81 \%)$ & - & 3 & - & - & - \\
\hline 3. & Pembrolizumab + erlotinib & $1(0.27 \%)$ & - & 1 & - & - & - \\
\hline 4. & Pembrolizumab + nivolumab + pemetrexed + ramucirumab & $1(0.27)$ & - & 1 & - & - & - \\
\hline 5. & Pembrolizumab + bevacizumab & $1(0.27)$ & - & 1 & - & - & - \\
\hline 6. & Pembrolizumab + gemcitabine & $1(0.27)$ & - & 1 & - & - & - \\
\hline 7. & Pembrolizumab + carboplatin + gemcitabine + necitumumab & $1(0.27)$ & - & 1 & - & - & - \\
\hline 8. & Pembrolizumab + afatinib & $1(0.27)$ & - & 1 & - & - & - \\
\hline 9. & Pembrolizumab + vinorelbine & $1(0.27)$ & - & - & 1 & - & - \\
\hline 10. & Pembrolizumab + bevacizumab + pemetrexed & $1(0.27)$ & - & - & 1 & - & - \\
\hline 11. & Pembrolizumab + bevacizumab + carboplatin + paclitaxel & $1(0.27)$ & - & - & - & 1 & - \\
\hline 12. & Pembrolizumab + docetaxel + ramucirumab & $1(0.27)$ & - & - & - & 1 & - \\
\hline 13. & Pembrolizumab + carboplatin + pemetrexed & $1(0.27)$ & - & - & - & 1 & - \\
\hline 14. & Pembrolizumab + nivolumab & $1(0.27)$ & - & - & - & 1 & - \\
\hline 15. & Pembrolizumab + paclitaxel protein-bound & $1(0.27)$ & - & - & - & - & 1 \\
\hline All $p$ & izumab-based regimens & $370(100.00)$ & 30 & 239 & 69 & 23 & 9 \\
\hline \multicolumn{8}{|c|}{ Atezolizumab-based regimens } \\
\hline 1. & Atezolizumab & $116(98.31)$ & - & 68 & 26 & 18 & 4 \\
\hline 2. & Atezolizumab + paclitaxel & $1(0.85)$ & - & - & 1 & - & - \\
\hline 3. & Atezolizumab + pemetrexed & $1(0.85)$ & - & - & 1 & - & - \\
\hline \multicolumn{2}{|c|}{ All atezolizumab-based regimens } & $118(100.00)$ & 0 & 68 & 28 & 18 & 4 \\
\hline
\end{tabular}

doses of pembrolizumab (median dose $2 \mathrm{mg} / \mathrm{kg}$ ) and three doses of atezolizumab. Median treatment duration with ramucirumab was 64 days for the Ram $\leftrightarrow \mathrm{ICI}$ cohort and 42 days for the Ram cohort. On the other hand, median treatment duration was 71 days with nivolumab, 44 days with pembrolizumab and 43 days with atezolizumab among the ICI cohort; and 85, 43 and 29 days, respectively, for the Ram $\leftrightarrow$ ICI cohort. Median duration of $1 \mathrm{~L}$ treatment among the Ram $\leftrightarrow \mathrm{ICI}$, Ram, and ICI cohorts was 93, 92 and 79 days, respectively.

Overall survival

Table 4 shows median OS estimates for the three treatment cohorts, overall and stratified by histology and duration 


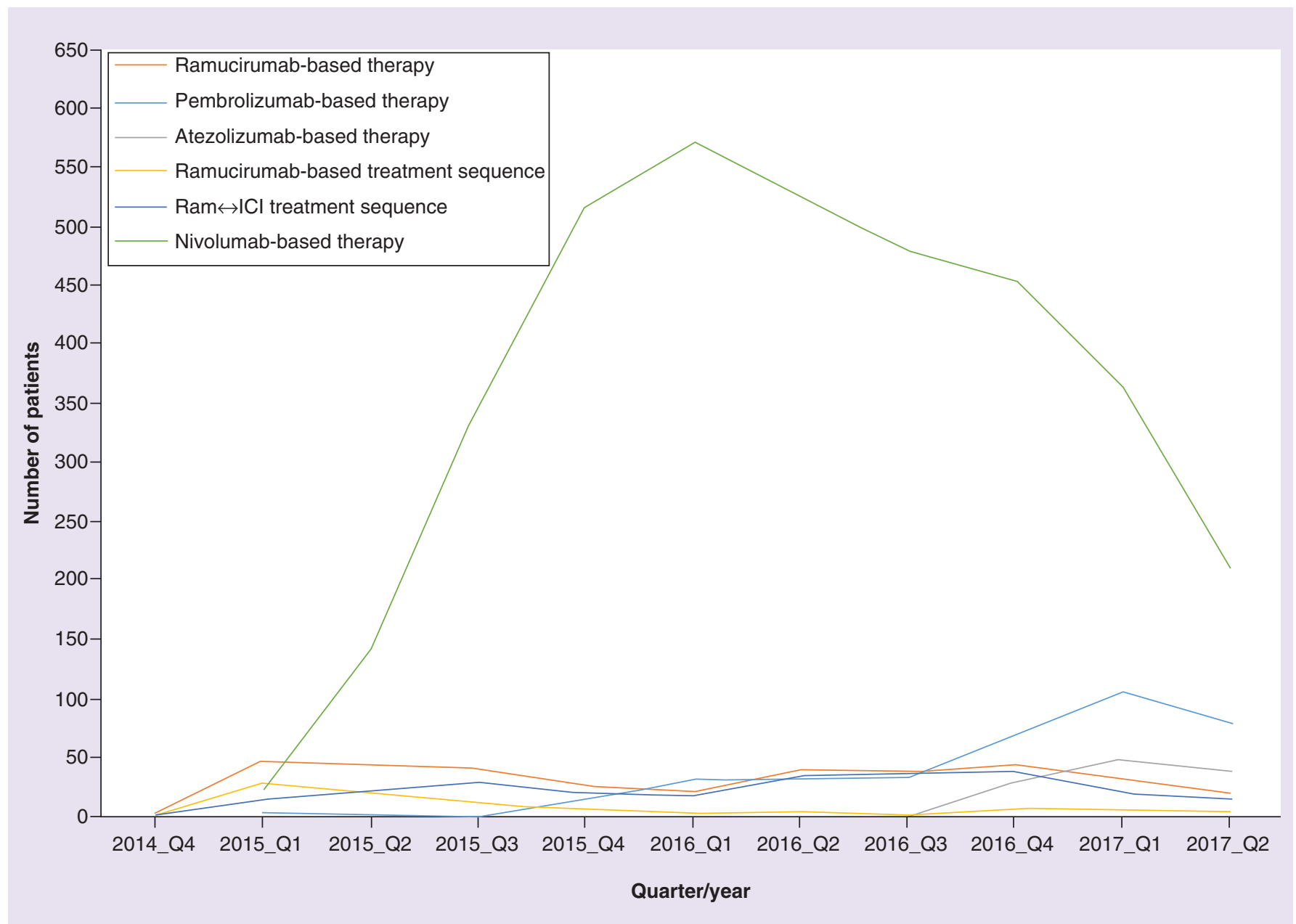

Figure 2. Trends for utilization of ramucirumab- or immune checkpoint inhibitor-based therapy in patients with advanced or metastatic non-small-cell lung cancer, by quarter.

ICI: Immune checkpoint inhibitor (nivolumab, pembrolizumab, atezolizumab); NSCLC: Non-small-cell lung cancer; Q: Quarter.

of 1L therapy. Overall, median OS (95\% CI) was 29.3 (25.5-33.0) months for the Ram $\leftrightarrow$ ICI cohort, 15.1 (12.6-18.2) months for the Ram cohort and 23.1 (21.9-24.2) months for the ICI cohort. Among the Ram $\leftrightarrow$ ICI cohort, median OS (95\% CI) was 31.4 (23.3-37.9) months for patients receiving ramucirumab- before ICI-based therapy and 26.5 (23.7-33.0) months for those treated with ramucirumab- after ICI-based therapy. For patients with limited ( $\leq 12$ weeks) duration on $1 \mathrm{~L}$ therapy and those with longer ( $>12$ weeks) duration, median OS (95\% CI) was, respectively, 23.8 (18.2-34.8) and 29.4 (26.1-37.9) months in the Ram $\leftrightarrow$ ICI cohort, 14.3 (8.8-19.7) and 15.5 (12.8-19.0) months in the Ram cohort, and 17.1 (15.9-18.3) and 28.2 (25.9-30) months in the ICI cohort.

\section{Discussion}

Taken together, findings from this analysis represent the first cohort study using real-world data from oncology practices across the USA to evaluate treatment patterns and outcomes for patients with aNSCLC treated with ramucirumab- or ICI-based therapy. As the optimal sequence, timing and combinations for postprogression treatment of aNSCLC have yet to be determined, increased understanding of how ramucirumab and ICIs are being used in routine clinical practice may provide important insights for healthcare decision makers, as well as generate hypotheses for future assessments on the utility of specific treatment sequencing strategies.

The OS findings in this study were directionally consistent with those from randomized trials showing relatively poor prognosis for patients with squamous histology [20-24] and those who discontinue 1L treatment after a short 
Ramucirumab-based treatment sequences

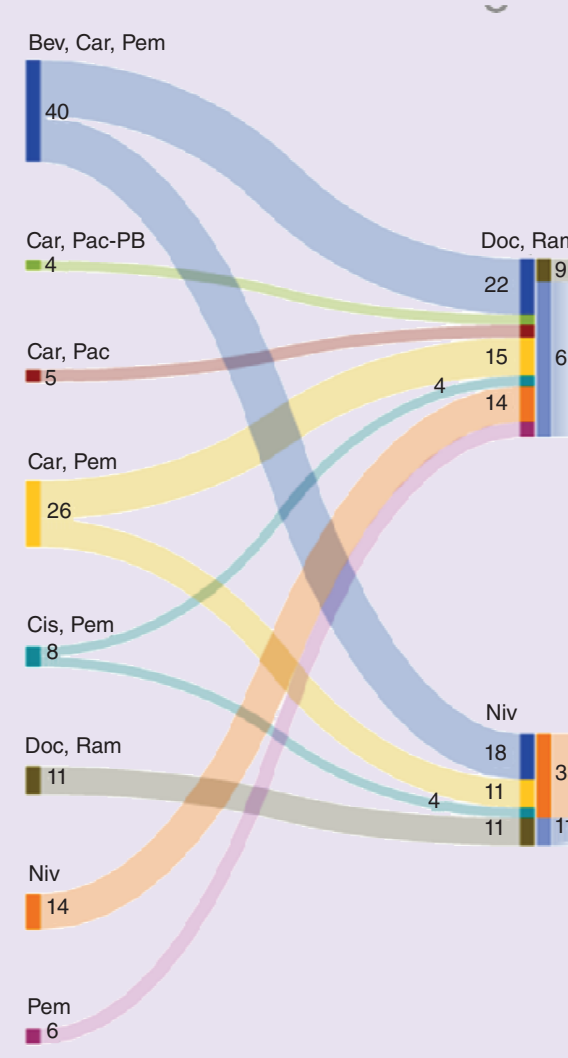

ICI-based treatment sequences

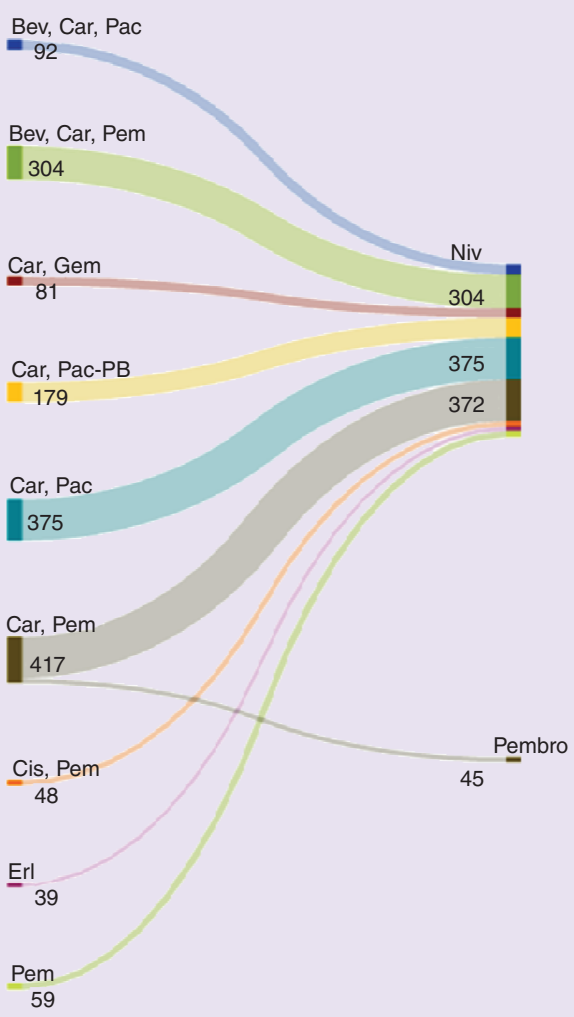

Figure 3. Sankey diagrams for the most common treatment sequences including ramucirumab- and/or immune checkpoint inhibitor-based therapy.

bev: Bevacizumab; car: Carboplatin; cis: Cisplatin; doc: Docetaxel; erl: Erlotinib; gem: Gemcitabine; ICl: Immune checkpoint inhibitor (nivolumab, pembrolizumab, atezolizumab); niv: Nivolumab; pac: Paclitaxel; pac-pb: Nab-paclitaxel; pem: Pemetrexed; pembro: Pembrolizumab; ram: Ramucirumab.

( $\leq 12$ weeks) duration [26]. However, the absolute survival estimates presented here should not be directly compared with those from clinical trials due to irreconcilable differences in design (e.g., lack of a control group), patient selection criteria and time frame between the studies. The index date for OS analysis in this study was the aNSCLC diagnosis date, whereas OS was indexed to initiation of postprogression treatment with ramucirumab + docetaxel or ICIs in the respective trials [20-24]. While the ramucirumab and ICI trial cohorts were predominantly male and less than 65 years of age, the current study cohort was roughly evenly split in gender, with a higher proportion of patients 65 years or older. Additionally, the current cohort had a higher percentage of black patients, whereas the respective trials had a higher proportion of white patients, with metastatic NSCLC. These and other (e.g., comorbidities, performance status) discrepancies between real-world and clinical trial populations have been reported previously [3133]. In contrast with clinical trials [20-24], unique combinations and sequences including ramucirumab or ICIs were observed in this study where treatment was selected at the discretion of the treating physician. The clinical rationale for this heterogeneity in treatment patterns and its implications on OS outcomes were not assessed, but possibly reflects the evolving treatment paradigm for NSCLC, especially in the past few years, and the complexity faced by clinicians regarding the optimal treatment choice and sequence of care for individual patients.

Although no formal comparative or trend analyses were conducted in this study, patients receiving ramucirumabbased therapy in sequential lines with ICI-based therapy, irrespective of whether ramucirumab-based therapy was used before or following ICI-based therapy, appeared to have numerically longer median OS than patients treated with sequencing strategies including one of the therapies and not the other. There are several possible explanations 
Table 3. Dosing and treatment duration with ramucirumab or immune checkpoint inhibitor therapy for patients with advanced or metastatic non-small-cell lung cancer.

\begin{tabular}{|c|c|c|c|c|c|c|c|c|}
\hline \multicolumn{2}{|c|}{ Cohort (n) } & \multicolumn{3}{|c|}{ Dose, $\mathrm{mg} / \mathbf{k g}$} & \multicolumn{2}{|c|}{ Treatment duration, days } & \multicolumn{2}{|c|}{ Number of cycles } \\
\hline Drug & $\begin{array}{l}\text { Number of } \\
\text { patients }\end{array}$ & $\begin{array}{l}\text { Number of } \\
\text { doses }\end{array}$ & Mean (SD) & $\begin{array}{l}\text { Median } \\
\text { (min-max) }\end{array}$ & Mean (SD) & $\begin{array}{l}\text { Median } \\
\text { (min-max) }\end{array}$ & Mean (SD) & $\begin{array}{l}\text { Median } \\
\text { (min-max) }\end{array}$ \\
\hline \multicolumn{9}{|l|}{ Overall cohort ${ }^{\dagger}$} \\
\hline - Ramucirumab & 357 & 1232 & $10(1.1)$ & $10(1-22)$ & $77(92.6)$ & $46(1-694)$ & $4(3.9)$ & $3(1-34)$ \\
\hline - Nivolumab & 3553 & 26,899 & $3(0.7)$ & $3(0.1-32)$ & $126(141.5)$ & $72(1-785)$ & $9(8.8)$ & $6(1-55)$ \\
\hline - Pembrolizumab & 363 & 1365 & $2(0.6)$ & $2(1-9)$ & $83(104)$ & $44(1-737)$ & $5(4.6)$ & $3(1-34)$ \\
\hline - Atezolizumab & 120 & 0 & - & - & $54(53)$ & $43(1-211)$ & $3(2.4)$ & $3(1-11)$ \\
\hline \multicolumn{9}{|l|}{ Ram $\leftrightarrow \mathrm{ICl}$ cohort ${ }^{\ddagger}$} \\
\hline - Ramucirumab & 245 & 910 & $10(1.2)$ & $10(1-22)$ & $84(94.1)$ & $64(1-694)$ & $5(4.2)$ & $4(1-34)$ \\
\hline - Nivolumab & 231 & 1747 & $3(0.4)$ & $3(1-5)$ & $125(114.4)$ & $85(1-674)$ & $9(7.2)$ & $6(1-43)$ \\
\hline - Pembrolizumab & 22 & 82 & $2(1)$ & $2(2-9)$ & $69(80.1)$ & $43(1-316)$ & $4(3.6)$ & $3(1-15)$ \\
\hline - Atezolizumab & 10 & 0 & - & - & $38(45.2)$ & $29(1-144)$ & $3(1.9)$ & $2(1-7)$ \\
\hline \multicolumn{9}{|l|}{ Ram cohort $\S$} \\
\hline - Ramucirumab & 112 & 322 & $10(1.1)$ & $10(1-13)$ & $63(88.1)$ & $42(1-498)$ & $3(2.9)$ & $3(1-16)$ \\
\hline \multicolumn{9}{|l|}{ ICl cohort $\mathbb{I}$} \\
\hline - Nivolumab & 3322 & 25152 & $3(0.7)$ & $3(0.1-32)$ & $126(143.2)$ & $71(1-785)$ & $9(8.9)$ & $6(1-55)$ \\
\hline - Pembrolizumab & 341 & 1283 & $2(0.6)$ & $2(1-5)$ & $84(105.4)$ & $44(1-737)$ & $5(4.7)$ & $3(1-34)$ \\
\hline - Atezolizumab & 110 & 0 & - & - & $55(53.6)$ & $43(1-211)$ & $3(2.4)$ & $3(1-11)$ \\
\hline \multicolumn{9}{|c|}{$\begin{array}{l}\text { †All eligible patients treated with ramucirumab- and/or ICI-based therapy in the treatment sequence. } \\
\text { ¥Patients treated with ramucirumab- and ICI-based therapy in the treatment sequence. } \\
\text { \& Patients treated with ramucirumab-based therapy, but not ICI-based therapy, in the treatment sequence. } \\
\text { I Patients treated with ICI-based therapy, but not ramucirumab-based therapy, in the treatment sequence. } \\
\text { ICI: Immune checkpoint inhibitor (nivolumab, pembrolizumab, atezolizumab); kg: Kilograms; mg: Milligrams; min: Minimum; max: Maximum; NSCLC: Non-small-cell lung cancer; SD } \\
\text { Standard deviation. }\end{array}$} \\
\hline
\end{tabular}

for this finding. First, observed OS results among the Ram $\leftrightarrow$ ICI, Ram and ICI cohorts, without adjustment for baseline risk (e.g., functional impairment, comorbidities and aggressiveness of disease) differences, could be driven by the diagnostic and clinical prognostic profile of patients, not sequencing strategies. Indeed, the current study suggests that physician-directed use of sequencing strategies including ramucirumab- and/or ICI-based therapy may be different for patients with different demographic or disease characteristics, including age, histology, initial stage at NSCLC diagnosis and PD-L1 expression. The Ram $\leftrightarrow \mathrm{ICI}$ cohort tended to be younger and more likely to have nonsquamous histology and Stage IIIB disease at initial diagnosis than the Ram and ICI cohorts, who more often had squamous histology and Stage IV disease at initial diagnosis. Second, current findings could be a reflection of how patients were assigned to study treatment cohorts. While all eligible patients received at least two lines of therapy, a larger proportion of the Ram $\leftrightarrow \mathrm{ICI}$ cohort received subsequent $(3 \mathrm{~L}+)$ therapy than the Ram or ICI cohorts, given the increased likelihood of use of ramucirumab- or ICI-based therapy in the postprogression setting during the study period. Of note, Ram or ICI cohorts were more often treated with standard chemotherapy in other lines not containing these regimens. As antiangiogenics and ICIs have individually demonstrated significant benefit over docetaxel in the postprogression setting [20-24], it is plausible that sequencing strategies including both treatment modalities would yield more benefit than those including just one of the modalities. While evidence to support the sequential use of antiangiogenics and immunotherapy is limited and warranted, preclinical and clinical data suggest that antiangiogenic therapy increases tumor sensitivity and response to immunotherapy [34]. Finally, observed OS findings could be due to a combination of these potential mechanisms. As sample size increases in real-world data, future research controlling for important baseline risk differences should evaluate the comparative survival outcomes of sequencing within the Ram $\leftrightarrow \mathrm{ICI}$ cohort to determine the optimal timing and sequencing of ramucirumab- relative to ICI-based therapy.

Treatment patterns observed in this study were mostly consistent with US labels for ramucirumab and ICIs [2730], recommendations from clinical guidelines [4,5] and prior observational studies describing national trends in use of systemic lung cancer treatment since 2015 [31,35,36]. For example, platinum-based chemotherapy and ICIs were the most commonly used regimens in the frontline and postprogression settings, respectively. The use of platinum in the postprogression setting was unexpected although this observation may be explained by the recent 


\begin{tabular}{|c|c|c|c|}
\hline Cohort & n & Median overall survival $(95 \% \mathrm{Cl})^{\dagger}$ & Percent censored \\
\hline \multicolumn{4}{|l|}{ Ram $\leftrightarrow \mathrm{ICl}$ cohort ${ }^{\ddagger}$} \\
\hline - Overall & 245 & $29.3(25.5-33.0)$ & $51 \%$ \\
\hline - Squamous & 59 & $24.1(17.7-29.4)$ & $49 \%$ \\
\hline - Nonsquamous & 177 & $31.4(26.0-35.7)$ & $51 \%$ \\
\hline - Ram pre-ICl${ }^{\S}$ & 83 & $31.4(23.3-37.9)$ & $43 \%$ \\
\hline - Ram post-ICII & 161 & $26.5(23.7-33.0)$ & $55 \%$ \\
\hline$-1 \mathrm{~L}$ treatment duration $\leq 12$ & 88 & $23.8(18.2-34.8)$ & $51 \%$ \\
\hline$-1 \mathrm{~L}$ treatment duration $>12$ & 145 & $29.4(26.1-37.9)$ & $52 \%$ \\
\hline \multicolumn{4}{|l|}{ Ram cohort ${ }^{\#}$} \\
\hline - Overall & 112 & $15.1(12.6-18.2)$ & $25 \%$ \\
\hline - Squamous & 20 & $15.1(9.2-27.3)$ & $30 \%$ \\
\hline - Nonsquamous & 90 & $15.5(12.6-18.3)$ & $24 \%$ \\
\hline$-1 \mathrm{~L}$ treatment duration $\leq 12$ & 48 & $14.3(8.8-19.7)$ & $23 \%$ \\
\hline$-1 \mathrm{~L}$ treatment duration $>12$ & 63 & $15.5(12.8-19.0)$ & $27 \%$ \\
\hline \multicolumn{4}{|l|}{ ICI cohort ${ }^{\dagger \dagger}$} \\
\hline - Overall & 3697 & $23.1(21.9-24.2)$ & $51 \%$ \\
\hline - Squamous & 1054 & $18.9(17.9-20.8)$ & $45 \%$ \\
\hline - Nonsquamous & 2492 & $25.8(24.2-28.0)$ & $54 \%$ \\
\hline$-1 \mathrm{~L}$ treatment duration $\leq 12$ & 1542 & $17.1(15.9-18.3)$ & $45 \%$ \\
\hline$-1 \mathrm{~L}$ treatment duration $>12$ & 1925 & $28.2(25.9-30.0)$ & $56 \%$ \\
\hline \multicolumn{4}{|c|}{$\begin{array}{l}\text { †Overall survival was defined as the time-period from the date of advanced or metastatic NSCLC diagnosis until death. } \\
\text { ¥Patients treated with ramucirumab- and ICI-based therapy in the treatment sequence. } \\
\text { § Patients treated with ramucirumab-based therapy followed by ICI-based therapy in the treatment sequence. } \\
\text { I Patients treated with ramucirumab-based therapy after ICI-based therapy in the treatment sequence. } \\
\text { \#Patients treated with ramucirumab-based therapy, but not ICI-based therapy, in the treatment sequence. } \\
\text { † Patients treated with ICI-based therapy, but not ramucirumab-based therapy, in the treatment sequence. } \\
\text { 1L: First-line; ICI: Immune checkpoint inhibitor (nivolumab, pembrolizumab, atezolizumab); NSCLC: Non-small-cell lung cancer. }\end{array}$} \\
\hline
\end{tabular}

approval and increasing use of pembrolizumab monotherapy in 1L [6,7]. The EGFR and $A L K$ testing rates were high, whereas testing rates were relatively low for $K R A S$ and $R O S 1$, as expected given the relative prognostic and predictive utility of these biomarkers in the tailored selection of NSCLC therapy. The PD-L1 testing rates were also low in this study, consistent with low usage of pembrolizumab, the only US FDA-approved ICI that requires selection of patients based on PD-L1 expression. It is of note, however, that pembrolizumab usage and PD-L1 testing rates observed in this study ( 9 and $24 \%$, respectively) are higher than those ( 3 and $8 \%$, respectively) reported by a previous study evaluating the use of ICIs in a US community practice cohort of aNSCLC patients [31], suggesting an increase in adoption of pembrolizumab and PD-L1 testing in this practice setting. Treatment with single-agent ramucirumab, in some cases following ramucirumab combination regimens, was expected as some patients in the REVEL trial who discontinued ramucirumab + docetaxel combination therapy were allowed to continue ramucirumab monotherapy [20]. The reuse of ramucirumab- or ICI-based regimens in sequential lines was observed in some patients, possibly suggesting favorable response with these regimens to justify retreatment by the treating physicians. Prior use of antiangiogenic (i.e., bevacizumab) or taxane therapy in patients treated with ramucirumab was not surprising as these patients benefited from ramucirumab + docetaxel therapy in REVEL [20]. At least one of every three patients treated with ramucirumab-based therapy in the present study was not naive to antiangiogenic therapy, which is more than double the $14 \%$ of patients who received $1 \mathrm{~L}$ bevacizumab prior to ramucirumab + docetaxel in REVEL [20].

While no formal tests were conducted to assess trends in usage of ramucirumab- or ICI-based therapy, the increasing use of sequencing strategies including ramucirumab- and ICI-based treatment starting in Q1 2015 is aligned with the rapid market uptake of ICIs in the postprogression setting since US FDA approval of nivolumab for NSCLC in March 2015 [16]. Given the observed increase in ICI use, relegation of ramucirumab-based treatment to $3 \mathrm{~L}$ after an ICI-based regimen in $2 \mathrm{~L}$ is conceivable, during this study time frame, as clinicians may believe that pushing back ramucirumab-based treatment by one or more lines to accommodate ICIs is a reasonable 
approach, especially in patients who overexpress PD-L1. Notably, 2L ramucirumab-based therapy following 1L ICI-based therapy (mostly nivolumab) was not uncommon, suggesting that clinicians may view antiangiogenic therapy as an appropriate treatment option following disease progression on initial therapy with ICI-based based regimens. Longer term data are needed to further understand these trends, given the rapidly evolving aNSCLC treatment landscape. As the standard of care in $1 \mathrm{~L}$ therapy shifts from chemotherapy alone to single-agent and/or combination immunotherapy [5-10], it is reasonable to expect increased adoption of sequencing strategies that include immunotherapy and antiangiogenics, as well as increased usage of ramucirumab-based therapies in earlier lines (e.g., $2 \mathrm{~L}$ vs $3 \mathrm{~L}$ ), particularly among patients who progress on or after $1 \mathrm{~L}$ immunotherapy + platinum-based chemotherapy.

There are limitations that must be considered when interpreting the results, including potential bias due to data entry and reporting errors, missing data, and unmeasured confounding. No comparative conclusions can be made based on the results as this analysis did not account for potential and observed imbalances between study groups in baseline prognostic factors, in the type and frequency of therapies received prior or subsequent to ramucirumabor ICI-based therapy, in the number of treatment lines received or in time intervals between aNSCLC diagnosis and initiation of treatment, which may have contributed to confounding and immortal time bias. In addition, the analysis did not account for the heterogeneity in drug combinations including ramucirumab or ICIs. Analysis of treatment duration and number of cycles did not adjust for censoring, which likely underestimated these measures. For OS analysis, censoring rates for ICI and Ram $\leftrightarrow$ ICI cohorts were high $(51 \%$ for both), which may have overestimated OS in these groups relative to the Ram cohort (censor rate, 25\%). In line with market uptake and usage trends, sample sizes were considerably smaller for cohorts treated with ramucirumab (Ram and Ram $\leftrightarrow$ ICI cohorts) than for the ICI cohort. Some important data elements used to characterize the eligible cohort (e.g., ECOG PS, comorbidities, level of PD-L1 expression (low vs high) were not available for all patients as there is limited standardization in collection of these variables in routine practice. Lastly, the data were drawn predominantly from community oncology centers and thus may not be generalizable to treatment patterns at academic medical centers. Given these limitations, survival outcome findings from the present study must be tempered and considered hypothesis-generating.

\section{Conclusion}

This analysis provides a real-world descriptive overview of OS for patients with aNSCLC treated with sequencing strategies including ramucirumab- and ICI-based therapies, as well as strategies including one of the therapeutic modalities, but not the other. Observed data suggest increasing usage of both modalities in sequential lines of therapy, including ramucirumab-following ICI-based therapies. These findings provide preliminary data for the design of future research to determine the best sequencing strategy for patients with aNSCLC.

\section{Future perspective}

With further development and adoption of single-agent and combination immunotherapy in the frontline setting for aNSCLC, clinicians will be faced with the task of determining the ideal sequence of therapy following disease progression on initial therapy. It is reasonable to expect increased utilization of sequencing strategies that include immunotherapy and antiangiogenics, as well as increased usage of ramucirumab-based therapies in earlier lines (e.g., $2 \mathrm{~L}$ vs $3 \mathrm{~L}$ ), particularly among patients who progress on or after $1 \mathrm{~L}$ immunotherapy + platinum-based chemotherapy. These changes in treatment patterns will likely further improve outcomes for patients with aNSCLC over the coming years.

\section{Previous presentation}

Data in this manuscript were in part disclosed at the ASCO Quality Care Symposium; March 2017; FL, USA.

Financial \& competing interests disclosure

This study was sponsored by Eli Lilly and Company, Indianapolis, IN, USA. C Molife, ZL Cui, XI Li, J Beyer, M Mahoui, and AB Oton are equity holders and employees of Eli Lilly and Company. LM Hess is an employee of Eli Lilly and Company. The authors have no other relevant affiliations or financial involvement with any organization or entity with a financial interest in or financial conflict with the subject matter or materials discussed in the manuscript apart from those disclosed. The authors have no other relevant affiliations or financial involvement with any organization or entity with a financial interest in or financial conflict with the subject matter or materials discussed in the manuscript apart from those disclosed. 


\section{Ethical disclosure}

This noninterventional study does not qualify as human subjects research in accordance with the US CFR, 45 CFR 46.102(f), and is thereby exempt from Institutional Review Board evaluation.

\section{Author contributions}

All authors attest to meeting the four ICMJE.org authorship criteria: substantial contributions to the conception or design of the work; or the acquisition, analysis or interpretation of data for the work; drafting the work or revising it critically for important intellectual content; final approval of the version to be published; and agreement to be accountable for all aspects of the work in ensuring that questions related to the accuracy or integrity of any part of the work are appropriately investigated and resolved.

\section{Open access}

This work is licensed under the Attribution-NonCommercial-NoDerivatives 4.0 Unported License. To view a copy of this license, visit http://creativecommons.org/licenses/by-nc-nd/4.0/

\section{Summary points}

- As the standard of care in frontline shifts from chemotherapy alone to single-agent and/or combination immunotherapy for advanced or metastatic non-small-cell lung cancer (aNSCLC), it has become increasingly important to understand the optimal treatment sequencing strategies when initial therapy fails.

- While randomized trials have shown survival benefits with immunotherapy or antiangiogenics over chemotherapy for advanced or metastatic aNSCLC, there is limited information on the effectiveness of sequencing strategies including these therapeutic modalities.

- This retrospective observational study described treatment patterns and overall survival (OS) for patients with aNSCLC who received at least two lines of therapy and were treated with systemic regimens containing ramucirumab (ram) or immune checkpoint inhibitors (ICls).

- Eligible patients were identified in the Flatiron Health electronic health record database and assigned to one of three treatment cohorts. The ram $(n=112)$ and ICI $(n=3697)$ cohorts included patients treated with sequences containing either ram- or ICl-based therapy, respectively, but not both. The Ram $\leftrightarrow \mathrm{ICl}$ cohort $(n=245)$ included patients who received both ramucirumab- and $\mathrm{ICl}$-based therapy in any sequence of care.

- Overall, ramucirumab-based therapy was most often (69\%) used in sequence with an ICl-based regimen, and of these patients, ram-based therapy was more frequently $(65 \%)$ used following ICl-based therapy. ICI-based regimens were most frequently used in sequential lines with chemotherapy.

- Both therapeutic modalities were mostly $(90 \%)$ used consistently with the respective US FDA-approved labels, and as expected, EGFR and $A L K$ testing rates were high whereas testing rates were relatively low for KRAS, ROS1 and PD-L1.

- The OS findings in this study were directionally consistent with those from clinical trials showing relatively poor prognosis for patients with squamous histology and those who discontinue first-line treatment after a short ( $\leq 12$ weeks) duration.

- Median OS (95\% Cl) was 29.3 (25.5-33.0) months for the Ram $\leftrightarrow \mathrm{ICl}$ cohort, 15.1 (12.6-18.2) months for the Ram cohort and 23.1 (21.9-24.2) months for the ICl cohort. These findings represent the first study to report real-world survival estimates for aNSCLC patients treated with sequencing strategies including ramucirumaband/or ICl-based therapies.

- While these data were from before immunotherapy was widely used in frontline, the data could provide a useful point of reference for healthcare decision makers regarding the effectiveness of sequential therapy with $\mathrm{ICI}$ and antiangiogenic therapies, for clinical practice benchmarking initiatives, and for future research as the NSCLC treatment landscape continues to evolve.

\section{References}

Papers of special note have been highlighted as: $\bullet \bullet$ of considerable interest

1. American Cancer Society: Cancer facts and figures 2018. (2018). www.cancer.org/content/dam/cancer-org/research/cancer-facts-and-st atistics/annual-cancer-facts-and-figures/2018/cancer-facts-and-figures-2018.pdf

2. American Cancer Society. About non-small cell lung cancer. (2018). www.cancer.org/content/dam/CRC/PDF/Public/8703.00.pdf

3. Noone AM, Howlader N, Krapcho M et al. SEER cancer statistics review, 1975-2015. (2018). https://seer.cancer.gov/csr/1975_2015/

4. Hanna N, Johnson D, Temin S et al. Systemic therapy for stage IV non-small-cell lung cancer: American Society of Clinical Oncology Clinical Practice guideline update. J. Clin. Oncol. 35, 3484-3515 (2017).

5. National Comprehensive Cancer Network. NCCN guidelines: non-small cell lung cancer version 3.2018. (2018). www.nccn.org/professionals/physician_gls/pdf/nscl.pdf 
6. Reck M, Rodríguez-Abreu D, Robinson AG et al. Pembrolizumab versus chemotherapy for PD-L1-positive non-small-cell lung cancer. N. Engl. J. Med. 375, 1823-1833 (2016).

-• Phase III randomized trial demonstrated significant improvement in survival with pembrolizumab versus platinum-based chemotherapy among treatment-naive patients with advanced non-small-cell lung cancer (NSCLC), PD-L1 expression on at least $\mathbf{5 0 \%}$ of tumor cells, and no $E G F R$ or anaplastic lymphoma kinase $(A L K)$ mutations.

7. Lopes G, Wu Y-L, Kudaba I, Kowalski D, Cho BC, Castro G. Pembrolizumab (pembro) versus platinum-based chemotherapy (chemo) as first-line therapy for advanced/metastatic NSCLC with a PD-L1 tumor proportion score (TPS) $\geq 1 \%$ : open-label, Phase 3 KEYNOTE-042 study [abstract]. J. Clin. Oncol. 36(18), LBA4 (2018).

-• Phase III randomized trial demonstrated significant improvement in survival with pembrolizumab versus platinum-based chemotherapy among treatment-naive patients with advanced NSCLC, PD-L1 expression on at least $1 \%$ of tumor cells, and no EGFR or $A L K$ mutations.

8. Gandhi L, Rodríguez-Abreu D, Gadgeel S et al. Pembrolizumab plus chemotherapy in metastatic non-small-cell lung cancer. $N$. Engl. J. Med. 378, 2078-2092 (2018).

-• Phase III randomized trial demonstrated significant improvement in survival with the addition of pembrolizumab to platinum-based chemotherapy among treatment-naive patients with metastatic nonsquamous NSCLC without $E G F R$ or $A L K$ mutations.

9. Paz-Ares L, Luft A, Vicente D et al. Pembrolizumab plus chemotherapy for squamous non-small-cell lung cancer. N. Engl. J. Med. doi: 10.1056/NEJMoa1810865 (2018) (Epub ahead of print).

-. Phase III randomized trial demonstrated significant improvement in survival with the addition of pembrolizumab to platinum-based chemotherapy among treatment-naive patients with metastatic squamous NSCLC.

10. Socinski MA, Jotte RM, Cappuzzo F et al. Atezolizumab for First-Line Treatment of Metastatic Nonsquamous NSCLC. $N$ Engl J Med. 378(24), 2288-2301 (2018).

11. Stinchcombe TE, Socinski MA. Current treatments for advanced stage non-small-cell lung cancer. Proc. Am. Thorac. Soc. 6, 233-241 (2009).

12. Arunachalam A, Li H, Bittoni MA et al. Real-world treatment patterns, overall survival, and occurrence and costs of adverse events associated with second-line therapies for medicare patients with advanced non-small-cell lung cancer. Clin. Lung Cancer 19, e783-e799 (2018).

13. Davis KL, Goyal RK, Able SL et al. Real-world treatment patterns and costs in a US Medicare population with metastatic squamous non-small cell lung cancer. Lung Cancer 87(2), 176-185 (2015).

14. Moro-Sibilot D, Smit E, de Castro Carpeño J et al. Outcomes and resource use of non-small-cell lung cancer (NSCLC) patients treated with first-line platinum-based chemotherapy across Europe: FRAME prospective observational study. Lung Cancer 88(2), 215-22 (2015).

15. Department of Health and Human Services, US Food and Drug Administration, 2014. (2014). www.accessdata.fda.gov/drugsatfda_docs/appletter/2014/125477Orig1s007ltr.pdf

16. Department of Health and Human Services, US Food and Drug Administration, 2015. (2015). www.accessdata.fda.gov/drugsatfda_docs/appletter/2015/125527Orig1s000ltr.pdf

17. Department of Health and Human Services, US Food and Drug Administration, 2015. (2015). www.accessdata.fda.gov/drugsatfda_docs/appletter/2015/125554Orig1s005ltr.pdf

18. Department of Health and Human Services, US Food and Drug Administration, 2016. (2016). www.accessdata.fda.gov/drugsatfda_docs/appletter/2016/125514Orig1s009ltr.pdf

19. Department of Health and Human Services, US Food and Drug Administration, 2016. (2016). www.accessdata.fda.gov/drugsatfda_docs/appletter/2016/761041Orig1s000ltr.pdf

20. Garon EB, Ciuleanu TE, Arrieta $\mathrm{O}$ et al. Ramucirumab plus docetaxel versus placebo plus docetaxel for second-line treatment of Stage IV non-small-cell lung cancer after disease progression on platinum-based therapy (REVEL): a multicentre, double-blind, randomised Phase 3 trial. Lancet 384, 665-673 (2014).

-. Phase III trial demonstrated significant improvement in survival with the addition of ramucirumab to docetaxel among platinum-treated patients with advanced NSCLC (all histologies).

21. Brahmer J, Reckamp KL, Baas P et al. Nivolumab versus docetaxel in advanced squamous-cell non-small-cell lung cancer. N. Engl. J. Med. 373, 123-135 (2015).

-• Phase III trial demonstrated significant improvement in survival with nivolumab versus single-agent docetaxel among platinum-treated patients with advanced squamous NSCLC.

22. Borghaei H, Paz-Ares L, Horn L et al. Nivolumab versus docetaxel in advanced nonsquamous non-small-cell lung cancer. $N$. Engl. J. Med. 373, 1627-1639 (2015).

-• Phase III randomized trial demonstrated significant improvement in survival with nivolumab versus single-agent docetaxel among platinum-treated patients with advanced nonsquamous NSCLC. 
23. Herbst RS, Baas P, Kim DW et al. Pembrolizumab versus docetaxel for previously treated, PD-L1-positive, advanced non-small-cell lung cancer (KEYNOTE-010): a randomised controlled trial. Lancet 387, 1540-1550 (2016).

-. Phase III trial demonstrated significant improvement in survival with pembrolizumab versus single-agent docetaxel among PD-L1 positive, platinum-treated patients with advanced NSCLC (all histologies).

24. Rittmeyer A, Barlesi F, Waterkamp D et al. Atezolizumab versus docetaxel in patients with previously treated non-small-cell lung cancer (OAK): a Phase III, open-label, multicentre randomised controlled trial. Lancet 389, 255-265.

-. Phase III trial demonstrated significant improvement in survival with atezolizumab versus single-agent docetaxel among platinum-treated patients with advanced NSCLC (all histologies).

25. Flatiron Health. https://flatiron.com/real-world-evidence/

26. Reck M, Paz-Ares L, Bidoli P et al. Outcomes in patients with aggressive or refractory disease from REVEL: a randomized Phase III study of docetaxel with ramucirumab or placebo for second-line treatment of stage IV non-small-cell lung cancer. Lung Cancer 112, 181-187 (2017).

27. Cyramza [package insert]. Indianapolis, IN: Eli Lilly and Company. (2018). http://pi.lilly.com/us/cyramza-pi.pdf

28. Opdivo [package insert]. Princeton, NJ: Bristol-Myers Squibb Company. (2018). https://packageinserts.bms.com/pi/pi_opdivo.pdf

29. Keytruda [package insert]. Whitehouse Station, NJ: Merck \& Company, Inc.. (2018). www.merck.com/product/usa/pi_circulars/k/keytruda/keytruda_pi.pdf

30. Tecentriq [package insert]. San Francisco, CA: Genentech, Inc. (2018). www.gene.com/download/pdf/tecentriq_prescribing.pdf

31. Khozin S, Abernethy AP, Nussbaum NC et al. Characteristics of real-world metastatic non-small cell lung cancer patients treated with nivolumab and pembrolizumab during the year following approval. Oncologist 23, 328-336 (2018). https://doi.org/10.1634/theoncologist.2017-0353

32. Khozin S, Blumenthal GM, Pazdur R. Real-world data for clinical evidence generation in oncology. J. Natl Cancer Inst. 109(11), 1-7 (2017).

33. Schilsky RL. Finding the evidence in real-world evidence: moving from data to information to knowledge. J. Am. Coll. Surg. 224, 1-7 (2017).

34. Manegold C, Dingemans AC, Gray JE. The potential of combined immunotherapy and antiangiogenesis for the synergistic treatment of advanced NSCLC. J. Thorac. Oncol. 12, 194-207 (2017).

35. Davies J, Patel M, Gridelli C, de Marinis F, Waterkamp D, McCusker ME. Real-world treatment patterns for patients receiving second-line and third-line treatment for advanced non-small cell lung cancer: a systematic review of recently published studies. PLoS ONE 12, 1-19, e0175679 (2017).

36. McKay C, Burke T, Cao X, Abernethy AP, Carbone DP. Treatment patterns for advanced non-small-cell lung cancer after platinum-containing therapy in U.S. community oncology clinical practice. Clin. Lung Cancer 17, 449-460 (2016). 